\title{
Postal Dosimetry Audits for the Domestic Medical Linear Accelerator
}

\author{
Kum Bae Kim ${ }^{1,2,3 \oplus}$, Sang Hyoun Choi ${ }^{1,20}$ \\ ${ }^{1}$ Research Team of Radiological Physics \& Engineering, Korea Institute of Radiological \& Medical Sciences, ${ }^{2}$ Department of Radiation \\ Oncology, Korea Institute of Radiological \& Medical Sciences, Seoul, ${ }^{3}$ Department of Medical Physics, Kyonggi University, Suwon, Korea
}

Received 19 May 2020

Revised 27 May 2020

Accepted 27 May 2020

Corresponding author

Sang Hyoun Choi

(sh524mc@gmail.com)

Tel: 82-2-970-1590

Fax: 82-2-970-1962
Purpose: The objective of this study is to perform Postal dosimetry audits for medical linear accelerators in radiation therapy institutions using glass dosimeters and Gafchromic film reading systems and postal dosimetry audit procedures, and to evaluate radiation therapy doses and mechanical accuracy in medical institutions.

Methods: Photon output measured and analyzed using a standard phantom for measuring photon output dose using a glass dosimeter for medical linear accelerators. Mechanical accuracy was measured and analyzed using software for film measurement.

Results: Measurement and analysis of photon beam output dose using a standard phantom glass dosimeter for photon beam output dose measurement was completed. All tolerance doses were within $5 \%$. Mechanical accuracy measurement and analysis using a standard phantom for verifying the mechanical accuracy of linear accelerator (LINAC) using a Gafchromic film were completed, and all results were shown within tolerances ( $2 \mathrm{~mm}$ or less).

Conclusions: In this study, Postal dosimetry audits were performed on the output dose and mechanical accuracy of photon beams (207 beams) for 106 LINACs from 48 institutions. As a result of corrective action and re-execution, it was confirmed that all engines met the acceptable standard within $2 \mathrm{~mm}$ in the linear accelerator.

Keywords: Postal dosimetry audit, Linear accelerator, Glass dosimeter, Gafchromic film

\section{Introduction}

In recent years, radiotherapy has been advancing toward achieving a higher cure rate with a higher therapeutic dose and minimum side-effects [1,2]. This has been possible through the development of high-performance and highprecision radiotherapy techniques and by applying cutting-edge medical technologies $[3,4]$. As of today, there are 182 medical linear accelerator (LINAC) machines in use for the radiotherapy of cancer patients in 97 radiotherapy facilities across the medical institutions in South Korea. The number goes up to 277 machines when CyberKnife,
Tomotherapy, GammaKnife, Vero, ViewRay, and Proton systems [5-10] are included. It is imperative to establish a system to measure the dose of medical radiation considering situations for which continuous maintenance of the equipment based on the establishment and verification of an optimized quality control system for LINAC and radiotherapy is required. To establish a medical radiationtraceable system between primary and secondary standard agencies and between secondary standard agencies and medical institutions, an external audit according to a regular and standardized procedure is critical. As a stabilized LINAC beam-quality factor should have an absolute effect 
on the improvement in radiotherapy performance due to its potential to apply the high-precision radiotherapy technique as well as the safety of medical radiation.

In many countries outside of South Korea, many attempts have been made to verify the reliability and accuracy of medical radiation.

Since 1969, the International Atomic Energy Agency (IAEA), a secondary standard dosimetry laboratory, in collaboration with the World Health Organization (WHO), has been conducting independent quality audits via mails, using thermoluminescence dosimetry (TLD) to verify the calibration of the teletherapy beam. The IAEA/WHO TLD program has verified the accuracy of more than 6200 photon-beam dosimeters in approximately 1500 hospitals for 37 years [11]. In Europe, the ESTRO-Quality Assurance network (EQUAL) has been run under Europe Against Cancer Program, in which the quality assurance project for radiotherapy has been performed using TLD from 1998 [12]. The Radiological Physics Center (RPC) in the United States has been providing quality audits for the institutions that participated in clinical studies collaborating with the National Cancer Institute (NCI) through the support of the NCI from 1968. Currently, it has changed its name to the Imaging and Radiation Oncology Core (IRCO), and has been operating various independent quality audit systems [13].

To maintain the dose accuracy of radiotherapy to the internationally recommended level, the Ministry of Food and Drug Safety in South Korea established its own dose assurance system in 1999 and provided the dose assurance and technical supports for photon beam and gamma rays of external radiotherapy machines using TLD from 2002 [14]. However, there have been no independent-quality audits in South Korea after the abolition of regulation on radiation dose assurance in 2009. Thereafter, the Internationally Accredited Calibration Laboratory in the Korea Institute of Radiological Medical Sciences (KIRAMS), with the support of the Korea Institute of Nuclear Safety, has been conducting independent quality audits for radiotherapy equipment from 2017.

The present study aimed to share methods and results of postal dosimetry audits conducted for output dose and mechanical accuracy of a photon beam (207 beams) in
2018, targeting 106 LINAC radiotherapy machines throughout South Korea.

\section{Materials and Methods}

\section{Calibration of glass dosimeter}

To evaluate the output dose of linear accelerators, a glass dosimeter (Asahi Techno Galss Corporation, Shizuoka, Japan) [15] was utilized, for which the following calibration factors were measured and then the difference was calibrated.

\section{1) Calibration of water-phantom}

For dosimetry of radiotherapy equipment, the radiation dose is mostly evaluated using the value measured in the water (the standard medium by using an ion chamber (the standard measuring device). Since postal dosimetry audits use plastic phantoms that have a density similar to that of water, it is necessary to calibrate the phantoms to accurately calculate and evaluate the absorbed dose to water. Thus, to obtain the calibration factor of the glass dosimeter for water, both water and the phantom were radiated with $200 \mathrm{MU}$, followed by calibration based on the difference.

\section{2) Calibration of the characteristics of each glass} dosimeter

Since the responses of glass dosimeters to radiation is different depending on lot and element, reading values differ to some extent even with the same dose. Therefore, to minimize this relative inaccuracy in measurement due to the characteristics of the glass dosimeter elements, the characteristics of each element should be calibrated. After irradiation of 1 Gy with the standard beam on every element for calibration of element characteristics, the reading value for each element was calibrated and then applied to dose evaluation. Each element was measured and read 10 times.

3) Calibration of energy dependency for glass dosimeter

Since different radiotherapy machine use different energy depending on the institution, leading to the difference 
in the energy radiated to glass dosimeters, it should be considered response characteristics of elements depending on photon beam energy. To calibrate such energy dependency of elements, the energy dependencies of the glass dosimeters were measured at 4, 6, 10, and 15 MV energy of the photon beam using a standard cobalt beam for calibration.

\section{4) Evaluation of output dose for each institution}

Considering calibration factors for dose calculation of the glass dosimeter as well as percent depth dose (PDD) or tissue maximum ratio (TMR) data for each institution, output doses of the glass dosimeter radiated in each institution were calculated. First, the glass dosimeter was read after irradiation at each institution, and then the count was converted to dose. To minimize the relative measurement uncertainty of each glass dosimeter and the reader, the count values of elements were obtained by applying three glass dosimeters thrice and then converting the mean value from a total of 90 counts to dose. After dose conversion, calibration factors for energy dependency, water-phantom, and individual characteristics of elements were applied, after which the final dose was calculated through an application of the calibration factors. Finally, the dose at the $\mathrm{d}_{\max }$ (maximum depth) was calculated from the calculated dose and PDD or TMR data of each institution, followed by comparison with the dose radiated from the institution at $\mathrm{d}_{\max }$.

\section{Fabrication of phantom}

\section{1) Fabrication of phantom for dosimetry}

To obtain a water-absorbed dose with the glass dosimeter, phantoms were fabricated by using water-equivalent plastic. acrylonitrile butadiene styrene copolymer has been used to fabricate the phantoms because it exhibits a relatively minor change in thickness due to its durability against shock and deliquescence in water (Fig. 1). The phantom was designed reflecting the recommendations of the American Association of Physicists in Medicine (AAPM) and IAEA TRS-398 as much as possible (ref). Regarding the use of phantoms to measure the dose, the phantoms were made in the size of $20 \mathrm{~cm} \times 20 \mathrm{~cm} \times 20 \mathrm{~cm}$ as per the guideline of making phantoms $5 \mathrm{~cm}$ larger than the field size to
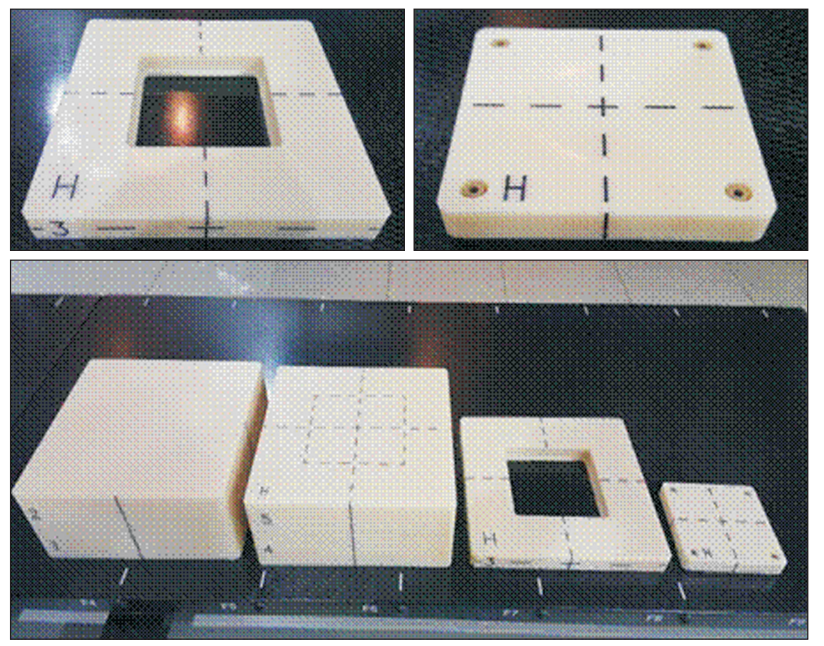

Fig. 1. Fabrication of phantom for measuring output dose using a glass dosimeter.

be measured. The glass dosimeter was placed $10 \mathrm{~cm}$ under the surface, considering the scattered ray when the phantom was installed.

\section{2) Fabrication and analysis of phantom for measurement of mechanical accuracy}

For evaluation of mechanical accuracy, it is measured the agreement of radiation field size as well as the accuracy of the central rotation axis for the gantry, couch and collimator, in which a total of four items were measured as a phantom set, and it was also designed to insert film holders for each item into the phantoms to minimize errors in measurements and analyses. Thus, the phantoms used for the measurement of mechanical accuracy were composed of a film holder for the four items and a phantom in which the film holder could be inserted. Fig. 2 presents the blueprint of the phantoms and the fabricated phantoms. To accurately align the central rotation axis in the radiotherapy machine, reticules were marked on both sides of the phantom.

The agreement of radiation field and various mechanical accuracies were measured and analyzed by using Gafchromic film (International Specialty Products, Wayne, NJ, USA) [16] and DoseLab software (Mobius Medical Systems, Houston, TX, USA) [17]. 

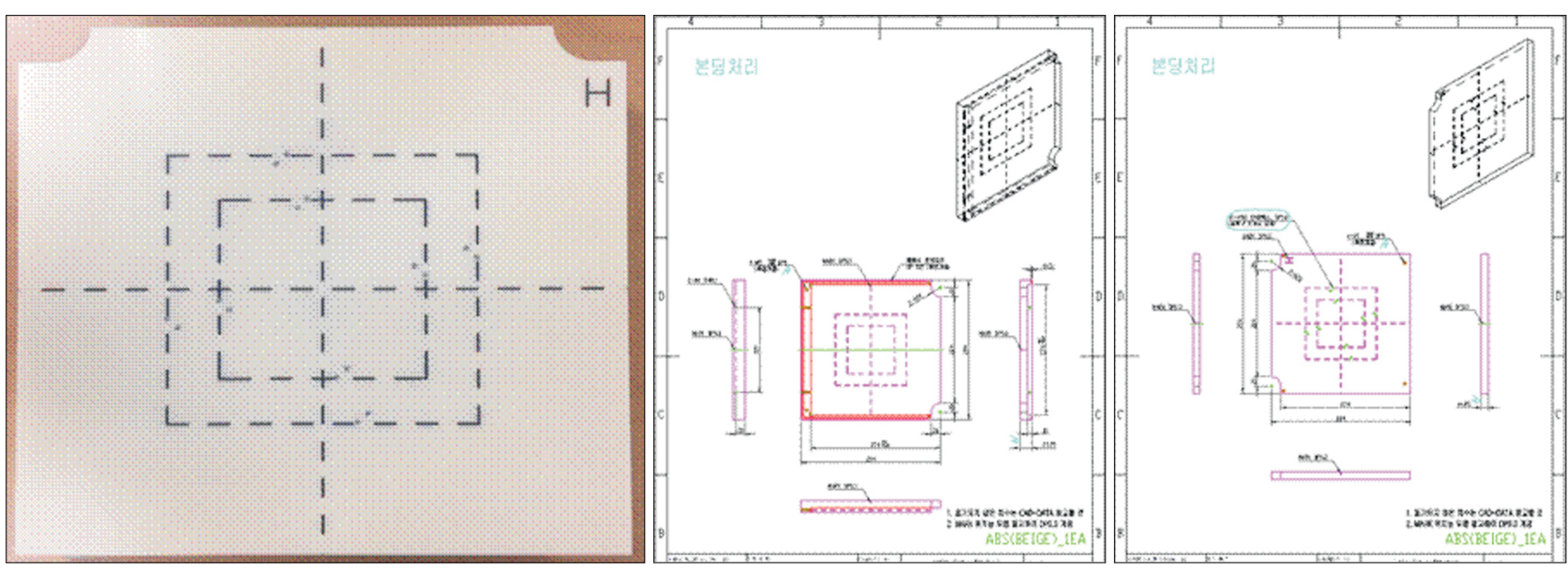

Fig. 2. Phantom for measuring mechanical accuracy.

Table 1. IAEA analysis-glass dosimeter irradiated by KIRAMS

\begin{tabular}{lccccc}
\hline \multirow{2}{*}{ Item } & \multicolumn{4}{c}{ Dose (irradiated) } \\
\cline { 2 - 6 } & $1.5 \mathrm{~Gy}$ & $1.75 \mathrm{~Gy}$ & $2 \mathrm{~Gy}$ & $2.25 \mathrm{~Gy}$ & $2.5 \mathrm{~Gy}$ \\
\hline Dose (measured) & $1.499 \mathrm{~Gy}$ & $1.749 \mathrm{~Gy}$ & $1.997 \mathrm{~Gy}$ & $2.252 \mathrm{~Gy}$ & $2.502 \mathrm{~Gy}$ \\
Analysis & $1.511 \mathrm{~Gy}$ & $1.752 \mathrm{~Gy}$ & $2.011 \mathrm{~Gy}$ & $2.260 \mathrm{~Gy}$ & $2.505 \mathrm{~Gy}$ \\
Difference & $0.8 \%$ & $0.2 \%$ & $0.8 \%$ & $0.3 \%$ & $0.1 \%$ \\
\hline
\end{tabular}

IAEA, International Atomic Energy Agency; KIRAMS, Korea Institute of Radiological Medical Sciences.

\section{Results}

\section{Comparative verification of glass dosimeters}

To assure the accuracy of radiation and analysis in glass dosimeters used for postal dosimetry audits, comparative verification was performed for glass dosimeters irradiated with 1.5-2.5 Gy together with the IAEA.

Table 1 presents the analysis results of glass dosimeters mailed after radiation in the KIRAMS. The dose in the parentheses was confirmed to be irradiated in the standard dosimeter calibrated by the Korea Research Institute of Standards and Science, based on which glass dosimeters were irradiated for the same period. The glass dosimeters were transferred to the IAEA along with three background elements. The sheet for analysis, supported by the IAEA, indicated that an element of each glass dosimeter showed as much as $10 \%$ of difference at $1.5 \mathrm{~Gy}$ and $2 \mathrm{~Gy}$, which were found to be the same with the reading results by the KIRAMS, and hence, such elements were excluded from the interpretation. Analysis results from each institution showed a sufficient accuracy $(<1 \%)$ of dose radiated in the KIRAMS, based on which it was found to have no problem in assuring the calibration coefficient for each ID of glass dosimeters. A second analysis was conducted in the IAEA, where 15 ( 5 doses $\times 3$ elements) glass dosimeters without radiation in the KIRAMS were sent after annealing and erasing, and then the IAEA radiated the corresponding dose, followed by interpretation in the KIRAMS.

Table 2 presents comparisons between the analysis results of glass dosimeters radiated in the IAEA and those in the Korea Institute of Radiological Medical Sciences. This comparison also showed that the difference in the reading values from the IAEA and the Korea Institute of Radiological Medical Sciences was small enough to be ignored $(<1 \%)$.

\section{Evaluation of output dose}

A postal dosimetry audit evaluated the output dose for 4 energy levels $(4,6,10,15 \mathrm{MV})$ used for each institution. The International Commission on Radiation Units and Measurements (ICRU) recommends a dose error for 
Table 2. KIRAMS analysis-glass dosimeter irradiated by IAEA

\begin{tabular}{cccccc}
\hline \multirow{2}{*}{ Item } & \multicolumn{3}{c}{ Dose (irradiated) } \\
\cline { 2 - 6 } & $1.5 \mathrm{~Gy}$ & $1.75 \mathrm{~Gy}$ & $2 \mathrm{~Gy}$ & $2.25 \mathrm{~Gy}$ & $2.5 \mathrm{~Gy}$ \\
\hline Anaysis & $1.493 \mathrm{~Gy}$ & $1.761 \mathrm{~Gy}$ & $1.986 \mathrm{~Gy}$ & $2.270 \mathrm{~Gy}$ & $0.9 \%$ \\
Difference & $0.5 \%$ & $0.6 \%$ & $-0.7 \%$ & $0.3 \%$ & $\mathrm{~Gy}$ \\
\hline
\end{tabular}

KIRAMS, Korea Institute of Radiological Medical Sciences; IAEA, International Atomic Energy Agency.
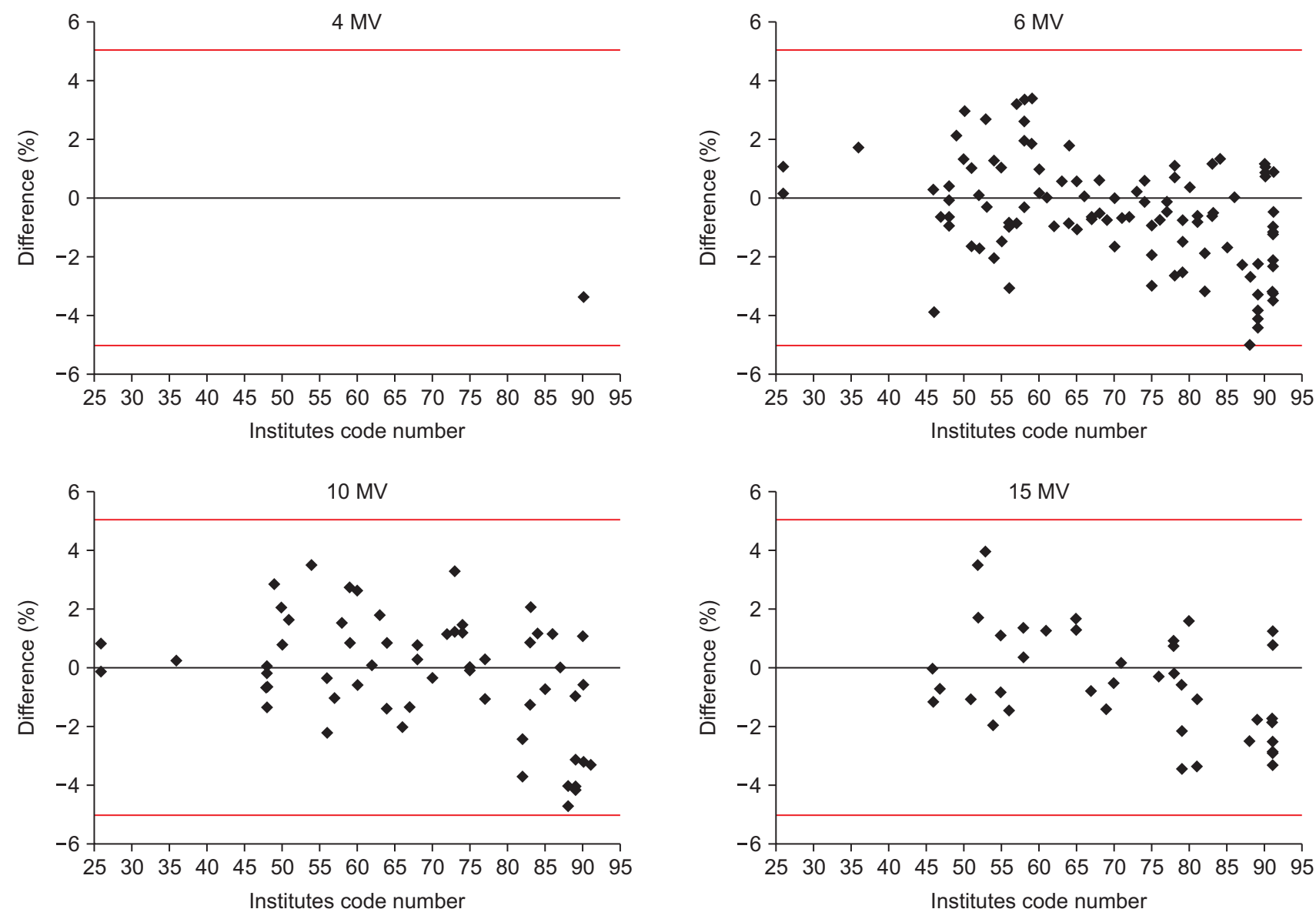

Fig. 3. Output dose result for 4, 6, 10, $15 \mathrm{MV}$ of linear accelerator.

radiotherapy of $\pm 5 \%$ as acceptable (ref). According to the postal dosimetry audit results, thus, an acceptable output dose level using glass dosimeters was set to $\pm 5 \%$, followed by evaluation for 4-, 6-, 10-, and 15-MV energy levels. As a result, output doses of all institutions were found to be within $\pm 5 \%$ (Fig. 3). Table 3 shows the statistics of evaluation for all institutions. The mean value represents its error for all institutions, along which the maximum, minimum, and standard deviation of the errors were presented. As for $4 \mathrm{MV}$, only 1 institution used it, and hence it was excluded from the statistical analysis of output doses.

Table 3. Evaluation results of linear accelerator 6, 10, $15 \mathrm{MV}$

\begin{tabular}{lccc}
\hline \multirow{2}{*}{\multicolumn{1}{c}{ Item }} & \multicolumn{3}{c}{ Energy (MV) } \\
\cline { 2 - 4 } & 6 & 10 & 15 \\
\hline Average (\%diff.) & -0.48 & -0.16 & -0.59 \\
Min. value (\%diff.) & -4.99 & -4.71 & -3.43 \\
Max value (\%diff.) & 3.86 & 3.50 & 3.93 \\
Standard deviation (\%) & 1.81 & 1.93 & 1.82 \\
\hline
\end{tabular}

\section{Evaluation of mechanical accuracy}

Fig. 4 shows the evaluation results of accuracy in radia- 


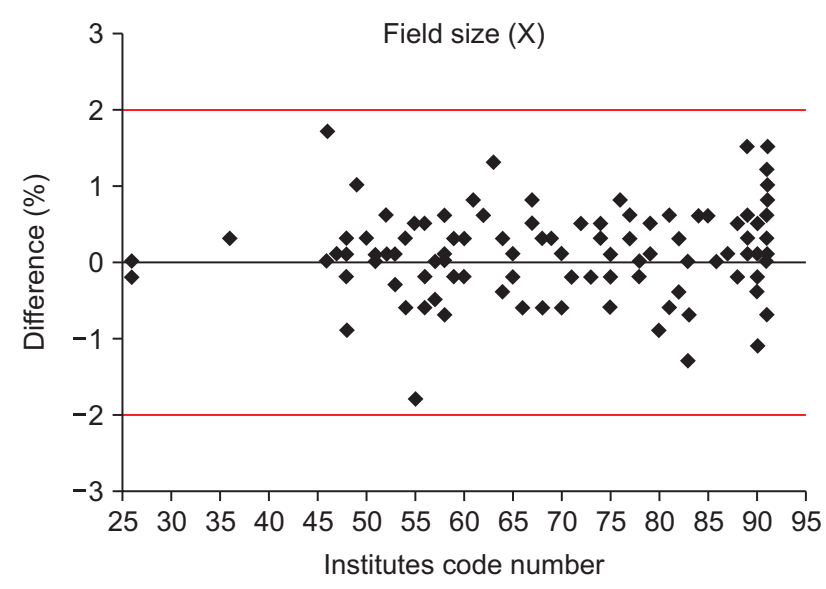

Fig. 4. Evaluation results for field size of linear accelerator.
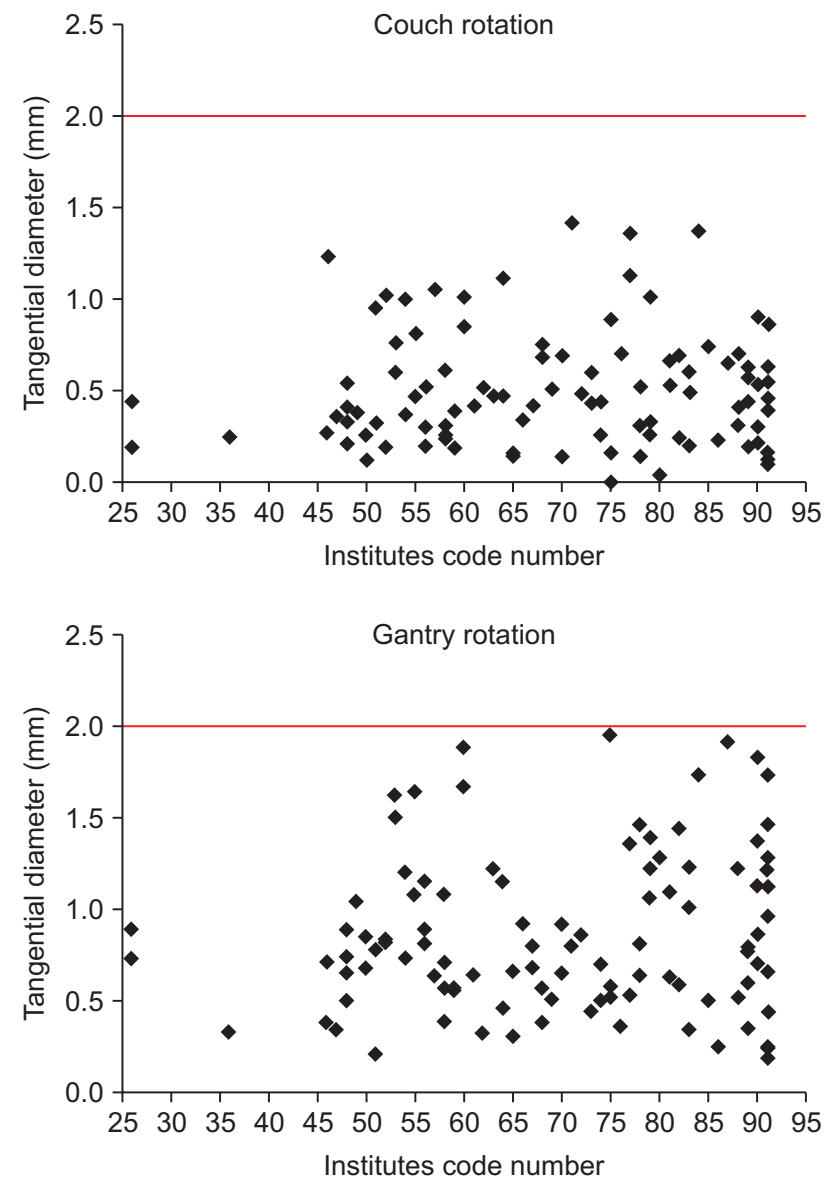

tion fields for all institutions. As the radiation field size was evaluated with $\pm 2 \mathrm{~mm}$ of acceptable error, the results for all institutions were found to be within the acceptable error range. The $\mathrm{X}$-axis of the radiation field size was between $-1.8 \mathrm{~mm}$ and $1.7 \mathrm{~mm}$ with a mean deviation of $0.11 \mathrm{~mm}$ and a standard deviation of $0.57 \mathrm{~mm}$, while the Y-axis of
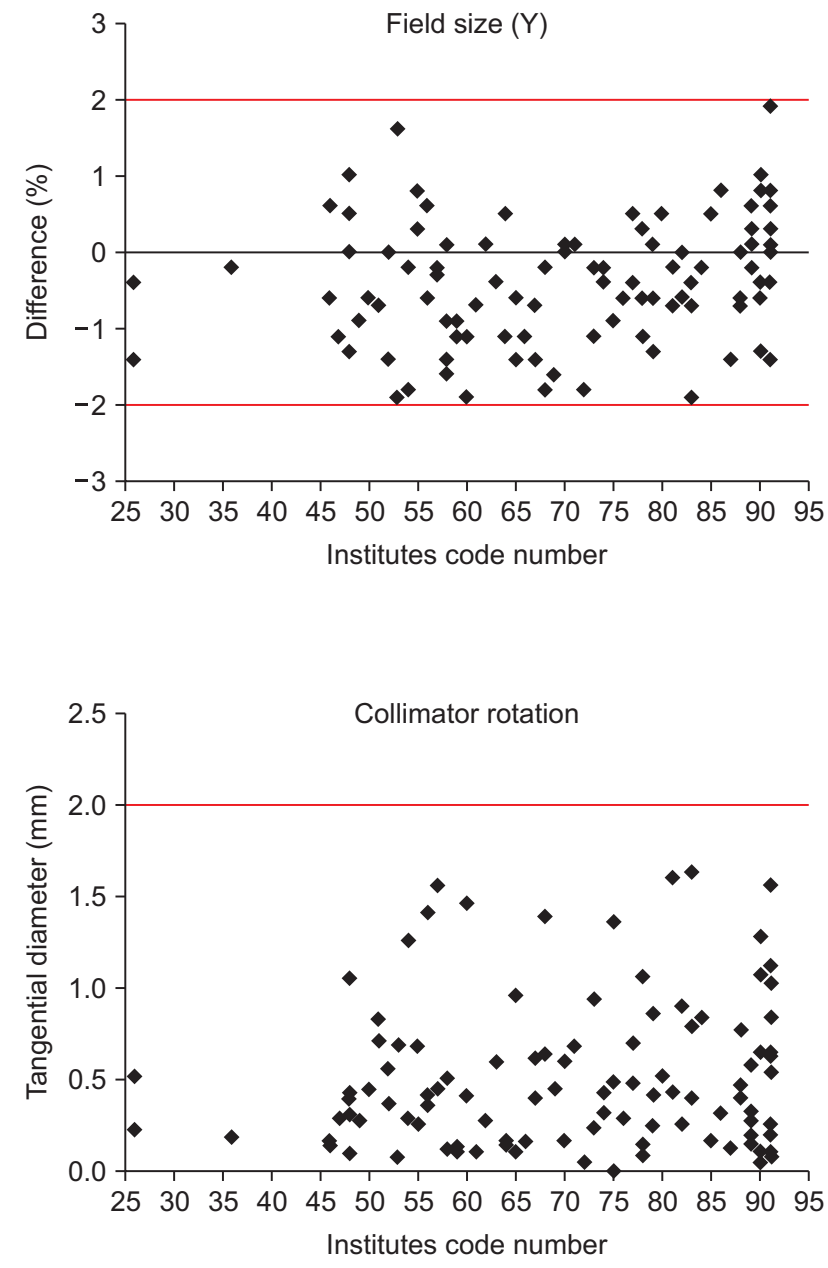

Fig. 5. Evaluation results of rotation center axis for couch, collimator, and gantry.

the field size was within $-1.9 \mathrm{~mm}$ to $1.9 \mathrm{~mm}$ with a mean deviation of $-0.4 \mathrm{~mm}$ and a standard deviation of $0.77 \mathrm{~mm}$. In addition, the evaluation on the central rotation axis for the couch, the collimator, and the gantry revealed that all institutions were within the acceptable level (Fig. 5). In the evaluation of the central rotation axis, the gantry showed 
the highest mean value $(0.85 \mathrm{~mm})$. Table 4 shows the statistics of the overall mechanical accuracy evaluation.

\section{Discussion}

\section{Statistical analysis of the results from postal dosimetry audits}

Fig. 6 shows the statistics of the evaluation results of the output dose by energy level in the machines of all the institutions. Of the 106 machines, 101 machines were using high energy, which was determined to have an optimal level except for 17 machines that were at acceptable levels. All 106 machines were using low energy, of which 85 and 16 machines were found to have optimal and acceptable levels, respectively.

Fig. 7 presents the statistics of the evaluation results on mechanical accuracy. All results on radiation field agreement as well as the accuracy of the central rotation axis in the couch, the collimator, and the gantry were within the optimal range. The central axis of the gantry was higher than those of the couch and collimator for Acceptable level.

\section{Analysis of the required period of postal dosimetry audits}

The mean duration of the audit for all the institutions was 11.6 days, which showed that many institutions either just met the recommended duration (10 days) or exceeded it (Table 5). Some institutions with early initiation could finish it within one day in addition to 2 days required for delivery/return, whereas most institutions were found to begin radiation about 3 days after checking the phantom.

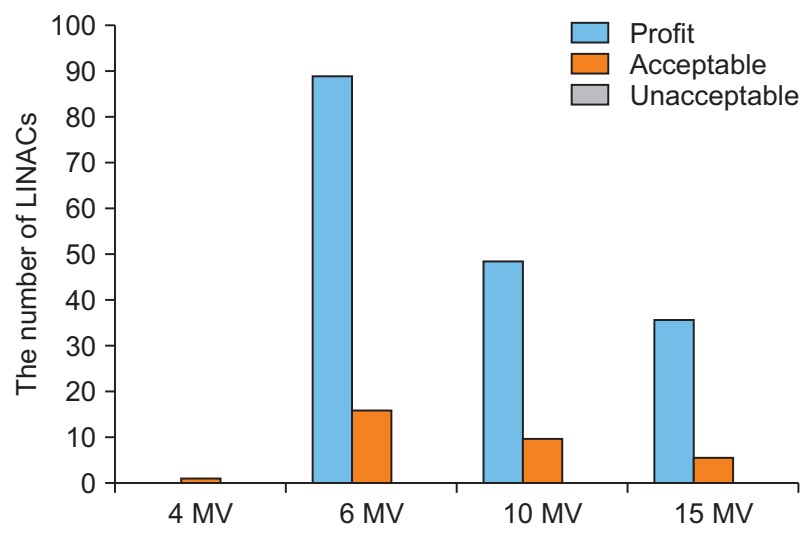

Fig. 6. Statistics of output dose evaluation by energy. LINAC, linear accelerator.

Table 4. Statistical values of the mechanical accuracy evaluation results

\begin{tabular}{|c|c|c|c|c|c|}
\hline Item & Field size (Y) & Field size (X) & Couch & Collimator & Gantry \\
\hline Average (diff.) & -0.40 & 0.11 & 0.49 & 0.52 & 0.85 \\
\hline Min. value (diff.) & -1.90 & -1.80 & 0.00 & 0.00 & 0.19 \\
\hline Max value (diff.) & 1.9 & 1.7 & 1.41 & 1.63 & 1.95 \\
\hline Standard deviation & 0.77 & 0.57 & 0.31 & 0.4 & 0.42 \\
\hline
\end{tabular}

Unit: $\mathrm{mm}$.
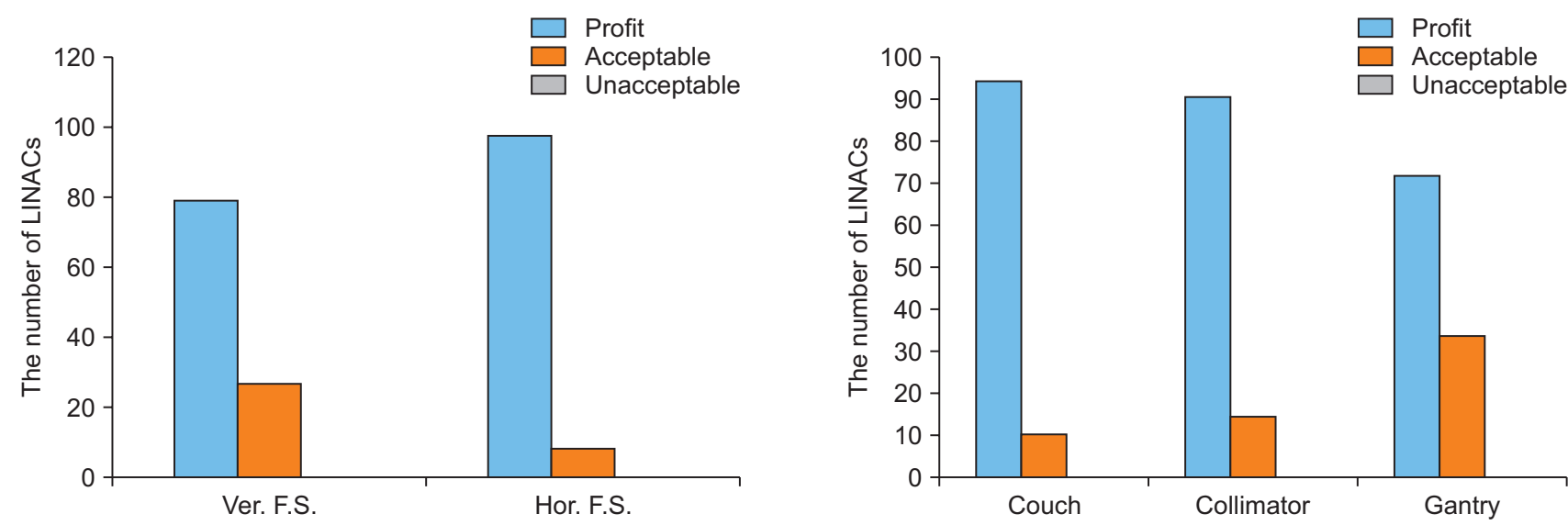

Fig. 7. Mechanical accuracy evaluation results. LINAC, linear accelerator. 
Table 5. Postal dosimetry audit lead time analysis

\begin{tabular}{lccc}
\hline \multicolumn{1}{c}{ Item } & 1st audit & Re-audit & Total time \\
\hline Average & 11.6 & 17.5 & 12.7 \\
Min. value & 0 & 5 & 0 \\
Max value & 47 & 82 & 103 \\
Standard deviation & 6.4 & 21.9 & 10 \\
\hline
\end{tabular}

Unit: day.

As shown in Fig. 8, an institution delayed the audit for up to 103 days while holding the phantom, during which some institutions were unable to begin the audit because they had no phantom. The institutions that took a long time for the audit were found to have a much larger accumulation of background values for the entire glass dosimeter elements than the other institutions, which might cause a small difference in interpretation.

\section{Errors in a postal dosimetry audit}

A detailed protocol for measurements of output dose and mechanical accuracy was enclosed to facilitate postal dosimetry audits. However, there were many cases of audit error due to the carelessness of the institutions and missing information, and thus a re-audit was performed along with the items that exceeded the acceptable range.

Of 48 institutions, 6 institutions took re-audit due to an error in measuring the output dose and exceeding the acceptable range. The most frequent error was due to the radiation emitted without changing the energy setting, while some errors were caused by not using the phantom. In addition, the highest number of re-audits for mechanical accuracy was caused by exceeding the acceptable range of the gantry isocenter tangent diameter, followed by exceeding the acceptable range of the field size. After re-audit, values improved.

\section{Conclusions}

The present study conducted postal dosimetry audits on output doses and mechanical accuracy of photon beams (207 beams), targeting 106 LINAC instruments in 48 institutions in South Korea. Regarding the output dose of the photon beam, re-audit was undertaken for 12 photon-

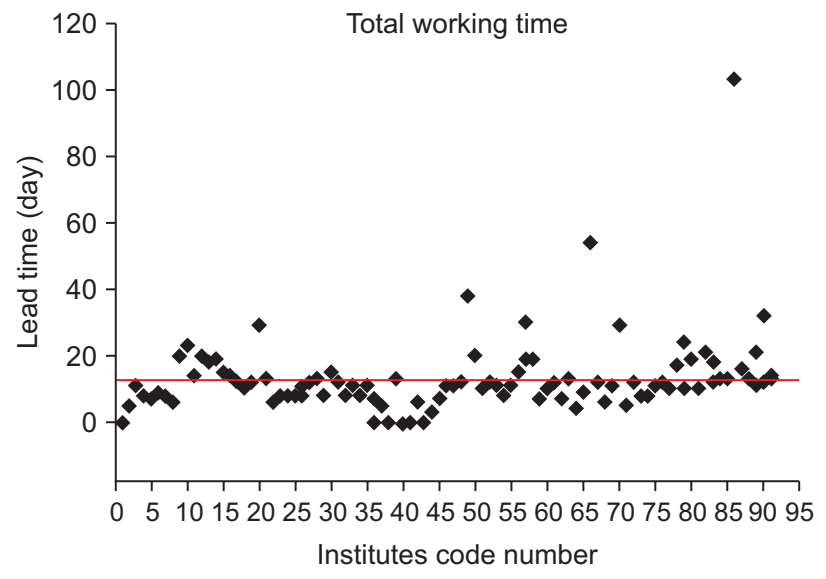

Fig. 8. Lead time conducted for postal dosimetry audit for all medical institutions.

beam energies of 10 institutions due to performance errors such as procedural and phantom set-up errors. As a result, all linear accelerators except for one instrument finally showed errors within $5 \%$ of the acceptable dose. The excluded institution was unable to be followed up due to the replacement of the corresponding old linear accelerator. Regarding mechanical accuracy, six linear accelerators in five institutions were subjected to re-audit due to exceeding the acceptable range of mechanical accuracy, while two institutions undertook re-audit due to the identification of the procedural error. In particular, some institution requested the manufacturer for a repair to correct an error of larger than $3 \mathrm{~mm}$ of the central rotation axis of the gantry, which was done eventually. Corrective measures and reaudit resulted in meeting the acceptance criteria within 2 $\mathrm{mm}$ in all linear accelerators of all institutions. In addition, peer reviews showed less than $1 \%$ of the difference, which assured the accuracy of the measurement system in postal dosimetry audit and reliability of the audit results. Additionally, a standard phantom should be designed for postal dosimetry audit to evaluate output dose and mechanical accuracy, targeting Tomotherapy and CyberKnife. Thus, previously established measurement and reading systems can be used properly for such radiotherapy machines.

\section{Acknowledgements}

This work was supported by the Creative Allied Project (CAP-15-06-ETRI) of the National Research Council of Sci- 
ence \& Technology.

\section{Conflicts of Interest}

The authors have nothing to disclose.

\section{Availability of Data and Materials}

All relevant data are within the paper and its Supporting Information files.

\section{Author Contributions}

Conceptualization, Data curation, Formal analysis, Funding acquisition, Investigation, Methodology, Project administration, Resources, Software, Supervision, Validation, Visualization, Writing - original draft, Writing-review \& editing: Kum Bae Kim, Sang Hyoun Choi.

\section{References}

1. Guerrero Urbano MT, Nutting CM. Clinical use of intensitymodulated radiotherapy: part I. Br J Radiol. 2004;77:88-96.

2. Syed YA, Patel-Yadav AK, Rivers C, Singh AK. Stereotactic radiotherapy for prostate cancer: a review and future directions. World J Clin Oncol. 2017;8:389-397.

3. Chin S, Eccles CL, McWilliam A, Chuter R, Walker E, Whitehurst $\mathrm{P}$, et al. Magnetic resonance-guided radiation therapy: a review. J Med Imaging Radiat Oncol. 2020;64: 163-177.

4. Glide-Hurst CK, Chetty IJ. Improving radiotherapy planning, delivery accuracy, and normal tissue sparing using cutting edge technologies. J Thorac Dis. 2014;6:303-318.

5. Mahboubi H, Sahyouni R, Moshtaghi O, Tadokoro K, Ghavami Y, Ziai K, et al. CyberKnife for treatment of vestibular schwannoma: a meta-analysis. Otolaryngol Head Neck Surg. 2017;157:7-15.

6. Saw CB, Katz L, Gillette C, Koutcher L. 3D treatment planning on helical tomotherapy delivery system. Med Dosim.
2018;43:159-167.

7. Aboukaïs R, Bonne NX, Touzet G, Vincent C, Reyns N, Lejeune JP. Progression of vestibular schawnnoma after GammaKnife radiosurgery: a challenge for microsurgical resection. Clin Neurol Neurosurg. 2018;168:77-82.

8. Verellen D, Depuydt T, Gevaert T, Linthout N, Tournel K, Duchateau M, et al. Gating and tracking, 4D in thoracic tumours. Cancer Radiother. 2010;14:446-454.

9. Ellefson ST, Culberson WS, Bednarz BP, DeWerd LA, Bayouth JE. An analysis of the ArcCHECK-MR diode array's performance for ViewRay quality assurance. J Appl Clin Med Phys. 2017;18:161-171.

10. Blanchard P, Gunn GB, Lin A, Foote RL, Lee NY, Frank SJ. Proton therapy for head and neck cancers. Semin Radiat Oncol. 2018;28:53-63.

11. Izewska J, Andreo P, Vatnitsky S, Shortt KR. The IAEA/ WHO TLD postal dose quality audits for radiotherapy: a perspective of dosimetry practices at hospitals in developing countries. Radiother Oncol. 2003;69:91-97.

12. Ferreira IH, Dutreix A, Bridier A, Chavaudra J, Svensson H. The ESTRO-QUALity assurance network (EQUAL). Radiother Oncol. 2000;55:273-284.

13. Sakhalkar H, Sterling D, Adamovics J, Ibbott G, Oldham M. Investigating the feasibility of 3D dosimetry in the RPC IMRT H\&N phantom. J Phys Conf Ser. 2009;164:12058.

14. Kim G, Oh H, Pyun W, Lee H. A quality assurance program for radiotherapy centers in Korea. Paper presented at: 22nd Annual International Conference of the IEEE; 2000 Jul 23-28; Chicago, USA. p. 1246-1249.

15. Rah JE, Oh DH, Kim JW, Kim DH, Suh TS, Ji YH, et al. Feasibility study of glass dosimeter for in vivo measurement: dosimetric characterization and clinical application in proton beams. Int J Radiat Oncol Biol Phys. 2012;84:e251-e256.

16. Robatjazi M, Mahdavi SR, Takavr A, Baghani HR. Application of Gafchromic EBT2 film for intraoperative radiation therapy quality assurance. Phys Med. 2015;31:314-319.

17. Huang JY, Pulliam KB, McKenzie EM, Followill DS, Kry SF. Effects of spatial resolution and noise on gamma analysis for IMRT QA. J Appl Clin Med Phys. 2014;15:4690. 